PRESENTACIÓN DE CASO

\title{
Perforación de colon sigmoides por espina de pescado: tratamiento combinado laparoscópico y endoscópico
}

\author{
Fishbone perforation of the sigmoid colon: combined laparoscopic and \\ endoscopic treatment
Cristina Magadán1, María Fidalgo1, Enrique Toledo1, José Manuel Olmos², Álvaro Terán², Juan Carlos Rodríguez-Sanjuan ${ }^{3}$, Patrizio Petrone ${ }^{4}$ \\ 1 Servicio de Cirugía General y del Aparato Digestivo, Hospital Universitario Marqués de Valdecilla, Santander, España \\ 2 Servicio de Aparato Digestivo y Endoscopias, Hospital Universitario Marqués de Valdecilla, Santander, España \\ 3 Jefe, Servicio de Cirugía General y del Aparato Digestivo, Hospital Universitario Marqués de Valdecilla, Santander, España \\ 4 Director of Surgical Research, Department of Surgery, NYU Winthrop Hospital, Mineola, Long Island, New York, USA. Clinical \\ Associate Professor of Surgery, New York Medical College, Valhalla, New York, USA
}

\section{Resumen}

La ingestión accidental de cuerpos extraños, más frecuentemente espinas de pescado, causa perforaciones intestinales en menos del I \% de los casos; la alta morbimortalidad asociada con estas ha favorecido la búsqueda de alternativas frente a la cirugía convencional.

El caso presentado muestra cómo el abordaje combinado secuencial laparoscópico y endoscópico, permite resolver con menor grado de agresión una perforación sigmoidea por cuerpo extraño.

Palabras clave: colon sigmoide; perforación intestinal; cuerpos extraños; diagnóstico; laparoscopía; endoscopía del sistema digestivo.

\begin{abstract}
Accidental ingestion of foreign bodies, more frequently fishbones, causes intestinal perforations in less than I\% of cases. The associated high morbidity and mortality has lead to the search for alternatives to conventional surgery.

The presented case shows how sequential approach, laparoscopic and endoscopic combined, allows a sigmoid perforation by a foreign body to be solved with a lower aggressive approach.
\end{abstract}

Key words: colon, sigmoid; intestinal perforation; foreign bodies; diagnosis; laparoscopy; endoscopy, digestive system.

Fecha de recibido: 5/12/2017 - Fecha aceptación: 25/05/2018

Correspondencia: Patrizio Petrone, MD, MPH, MHSA, FACS, Director of Surgical Research Program Director, International Visiting Scholars \& Research Fellowship, Department of Surgery - NYU Winthrop Hospital, 222 Station Plaza North, Suite 603, Mineola, Long Island, New York 11501 - USA

Correo electrónico: patrizio.petrone@gmail.com - patrizio.petrone@nyulangone.org

Citar: Magadán C, Fidalgo M, Toledo E, Olmos JM, Terán A, Rodríguez-Sanjuán JC, et al. Perforación de colon sigmoides por espina de pescado: tratamiento combinado laparoscópico y endoscópico. Rev Colomb Cir. 2018;33:433-7. https://doi.org/10.30944/20117582.91

Este es un artículo de acceso abierto bajo una Licencia Creative Commons - BY-NC-ND https://creativecommons.org/licenses/by-nc-nd/4.0/deed.es 


\section{Introducción}

La ingestión accidental de cuerpos extraños es una urgencia común. La mayoría recorre el tubo digestivo sin complicaciones en un tiempo variable (días a semanas) ${ }^{\mathrm{I}-3}$, aunque en Io a $20 \%$ de los casos se requiere su extracción endoscópica ${ }^{2,4}$. La perforación gastrointestinal ocurre en menos del I \% de los casos ${ }^{\mathrm{I}-3,5}$, sin embargo, este porcentaje aumenta hasta I5 a $35 \%$ si el objeto ingerido es punzante ${ }^{6}$.

Existe mayor riesgo de ingestión de un cuerpo extraño, y por lo tanto de perforación, en la población infantil, enfermos mentales, ancianos, alcohólicos y en quienes usan prótesis dental ${ }^{3}$, así como en aquellos con una enfermedad intestinal intrínseca. Las espinas de pescado son el objeto más frecuentemente encontrado, en relación proporcional con la incorporación de este alimento en la dieta.

La primera publicación registrada sobre este tipo de perforación, data de $184 I^{5}$. No obstante, el abordaje combinado, quirúrgico y endoscópico, apenas aparece referenciado en la literatura.

La presentación es tan variable como la posibilidad de perforación del tubo digestivo, por lo que forma parte del diagnóstico diferencial de enfermedades tan frecuentes como la apendicitis, la úlcera péptica y la diverticulitis aguda ${ }^{5}$, y puede cursar de manera aguda, subaguda o crónica.

Las manifestaciones clínicas son inespecíficas ${ }^{7} \mathrm{e}$ incluyen fiebre, afectación del estado general y dolor abdominal localizado o difuso, dependiendo fundamentalmente de la localización anatómica, la gravedad y el tiempo de evolución 5. Menos frecuente es la presencia de obstrucción intestinal o hemorragia ${ }^{\mathrm{I}}$.

La formación de abscesos puede dar lugar a hidronefrosis obstructiva de causa mecánica. La perforación anorrectal puede favorecer la presencia de sepsis perianal y gangrena de Fournier ${ }^{7}$. Presenta una considerable morbimortalidad, que se asocia con la presencia de absceso intraabdominal, tumoración de pared, fístula intestinal, pseudotumores, bacteriemia, o peritonitis localizada o difusa ${ }^{5}$; además, pue- de migrar hacia órganos adyacentes, como el bazo $^{2}$ y el hígado ${ }^{8}$.

Se afecta con mayor frecuencia $(73-83 \%)^{1,7} \mathrm{el}$ segmento ileocecal ${ }^{9}$, seguido por el duodeno ', coincidiendo con áreas de estrechez anatómica; también, se pueden afectar el esófago, el píloro, el ano y, más infrecuentemente, la unión rectosigmoidea, como se describe en la literatura 5,9 .

Se han descrito formas de presentación atípicas, como la perforación del apéndice cecal ${ }^{\mathrm{I}}$, la perforación de un divertículo de $\mathrm{Meckel}^{\mathrm{IO}} \mathrm{o}$ la ruptura esplénica ${ }^{2}$.

La tomografía computadorizada (TC) sin contraste es el método diagnóstico de elección, puesto que permite filiar la etiología, la localización y las complicaciones asociadas; los hallazgos más frecuentes son el engrosamiento de la pared intestinal, la estriación de la grasa adyacente o el absceso localizado, y el menos común, la presencia de aire libre "I. Infortunadamente, el diagnóstico preoperatorio puede ser dificultoso, por lo que la ausencia de contraste oral y endovenoso ayuda a identificar aumentos de densidad que, de otra manera, podrían pasar inadvertidos. La radiografía simple de abdomen puede resultar de utilidad para identificar neumoperitoneo hasta en $30 \%$ de los casos ${ }^{1,5}$.

Algunos estudios reportan que entre 80 y $90 \%$ de las perforaciones por espina de pescado, se diagnostican durante la exploración quirúrgica ${ }^{6}$.

\section{Caso clínico}

Se trata de un varón de 76 años de edad, con dislipidemia en tratamiento con atorvastatina, sin otros antecedentes de interés ni intervenciones quirúrgicas previas. Acudió a urgencias por presentar dolor abdominal de inicio súbito en la fosa ilíaca izquierda, aproximadamente, de 12 horas de evolución.

A la exploración, presentaba distensión abdominal con reacción peritoneal local en la fosa ilíaca izquierda, febrícula y signos vitales estables. En los exámenes de laboratorio, se destacan leucocitosis y neutrofilia, sin formas inmaduras. Refirió ingestión de pescado en las últimas 48 horas. 
En la TC se observó un cuerpo extraño (espina de pescado) incrustado en el tercio distal del colon descendente, con escasas burbujas de gas libre y diverticulosis del colon (figura I).

Se inició antibioterapia empírica y se decidió practicar una laparoscopia exploradora, consensuado con el paciente. Se encontró un plastrón local y material purulento circundante que fue drenado. En la pared sigmoidea, se apreció engrosamiento focal, sin visualizarse el cuerpo extraño, que no atravesaba completamente la pared del colon. Se hicieron lavados y se colocó un drenaje adyacente a la zona afecta.

En las siguientes 24 horas, se practicó una endoscopia digestiva baja y se extrajo una espina de pescado de $3,8 \mathrm{~cm}$ de longitud, sin complicaciones inmediatas (figuras 2 y 3 ).

El periodo posoperatorio transcurrió sin incidencias, salvo por un íleo adinámico que se manejó de forma conservadora. Se retiró el drenaje intraabdominal, que fue escaso y de características normales, en las primeras 72 horas tras iniciarse el tránsito intestinal. No se observaron otras complicaciones durante el seguimiento a largo plazo.

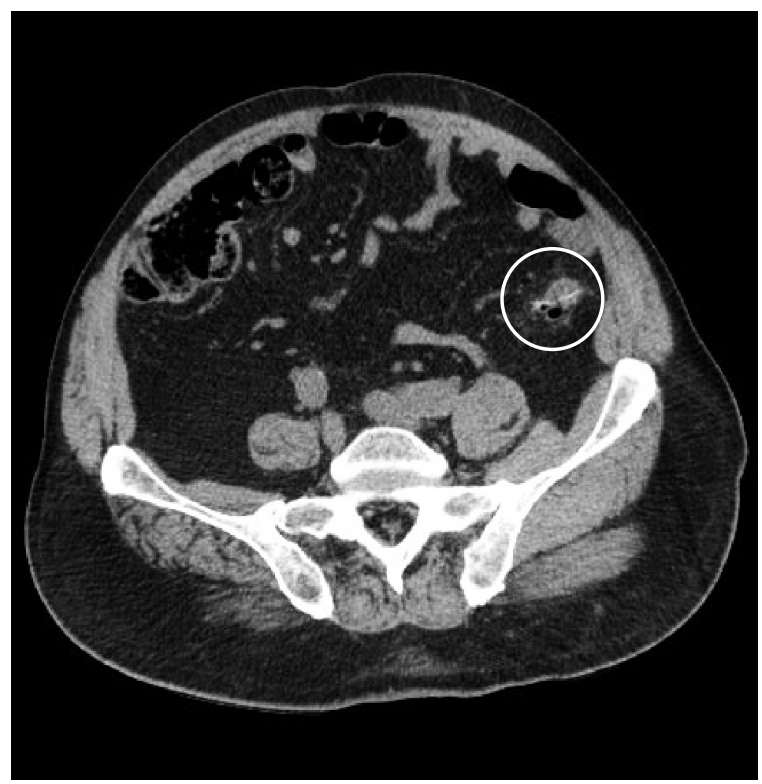

Figura 1. Se observa dentro del círculo un cuerpo extraño impactado en el tercio distal del colon descendente.

\section{Discusión}

Después de una adecuada reanimación hidroelectrolítica, el objetivo del tratamiento consiste en un adecuado control del foco infeccioso, la reducción de la contaminación bacteriana mediante la extracción del cuerpo extraño y la prevención de la infección recurrente o persistente 5.

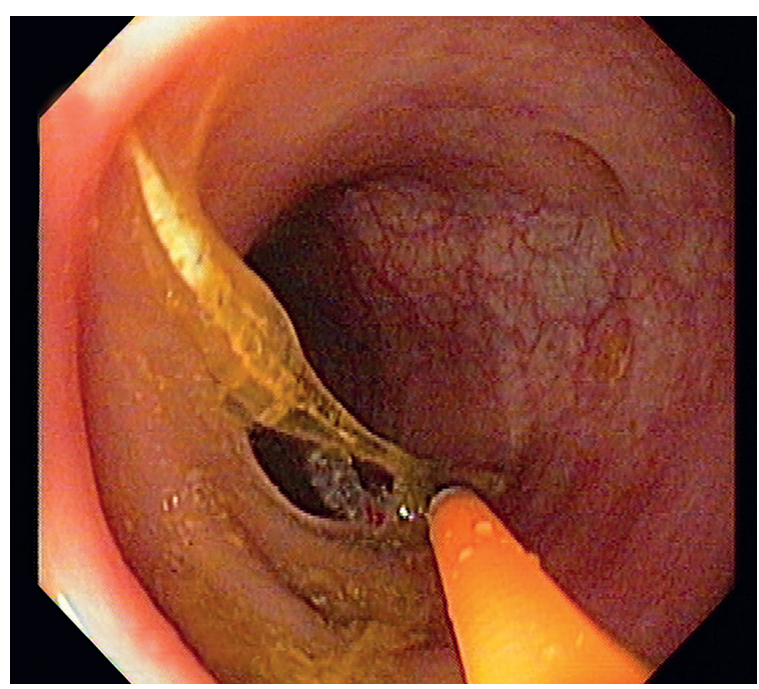

Figura 2. Endoscopia digestiva baja para extraer una espina de pescado

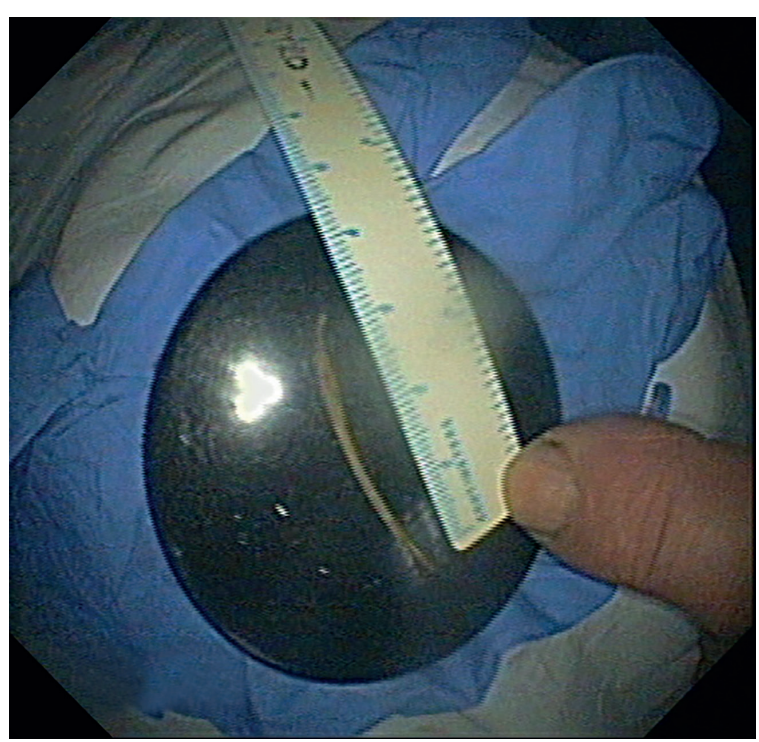

Figura 3. Cuerpo extraño (espina de pescado) extraída por endoscopia digestiva baja 
Clásicamente, se ha planteado el abordaje quirúrgico abierto como la única alternativa para el control del foco infeccioso, mediante el cierre primario o la resección del segmento afecto. Esta intervención se asocia más frecuentemente con la creación de un estoma que con una anastomosis primaria, lo cual se justifica por el proceso inflamatorio, por lo que la morbimortalidad descrita en la literatura es importante 5 . Durante la cirugía, se deben descartar fístulas o perforaciones en las áreas adyacentes ${ }^{1}$.

Recientemente, se ha sugerido un manejo conservador inicial en casos seleccionados. Sin embargo, la intervención quirúrgica puede ser necesaria si el objeto permanece durante más de una semana, se mantiene en la misma posición durante tres días o se produce un empeoramiento del estado general ${ }^{6}$.

El abordaje endoscópico aislado ha sido muy empleado, fundamentalmente para la extracción de cuerpos extraños mediante endoscopia digestiva alta, cuando no se presenta paso espontáneo durante las primeras 24 horas tras la ingestión ${ }^{3}$. Los objetos de más de $5 \mathrm{~cm}$ se asocian con complicaciones como sangrado, incrustación, obstrucción o perforación ${ }^{4}$, mientras que el empleo del endoscopio flexible se asocia con un menor riesgo de perforación ${ }^{3}$.

Más infrecuente es su utilización en perforaciones gastrointestinales altas (fundamentalmente duodenales); se pueden usar clips para el cierre primario ${ }^{4,6}$. Se considera seguro el manejo endoscópico en ausencia de contaminación peritoneal demostrada ${ }^{7}$.

La laparoscopia exploradora es un procedimiento que se debe considerar en caso de duda diagnóstica. Tras la confirmación, se debe considerar la conversión a cirugía abierta, aunque no siempre resulta necesaria ?

La intervención puede ser dificultosa durante la fase aguda; por esta razón, en casos seleccionados, se plantea un manejo inicial con antibioterapia de amplio espectro y diferir la cirugía hasta repetir la TC, para confirmar la ausencia de migración y descartar otras complicaciones ${ }^{7}$.
El abordaje combinado, laparoscópico y endoscópico, ha sido escasamente descrito en la literatura. Sin embargo, su empleo está justificado en perforaciones asociadas a afectación del peritoneo visceral, pues permite controlar el foco infeccioso, retirar el cuerpo extraño y manejar las complicaciones asociadas o potenciales, con un menor grado de agresión.

En el presente caso, y teniendo en cuenta el contexto clínico, la perforación parece estar relacionada con la incrustación de la mencionada espina de pescado, al tratarse de un paciente sin alteraciones previas del ritmo intestinal y, prácticamente, sin comorbilidades ni tratamientos de base que hicieran pensar en una perforación espontánea.

El abordaje mínimamente invasivo combinado que se utilizó, permitió lograr estos objetivos mediante la aspiración del material fibrino-purulento, la extracción del agente causal y la colocación de drenaje intraabdominal, tras un intento inicial infructuoso de manejo conservador.

\section{Conclusiones}

El manejo combinado, endoscópico y laparoscópico, es una opción factible y segura, en casos seleccionados de perforación contenida o peritonitis localizada por la incrustación del cuerpo extraño en el colon, uniendo los beneficios de la cirugía mínimamente invasiva y la endoscopia.

\section{Referencias}

I. Beh JCY, Uppaluri AS, Koh BFJ, Cheow P. Fishbone perforated appendicitis. J Radiol Case Rep. 20I6;IO:I422. doi: I0.394I/jrcr.vioi7.2826

2. Sierra-Ruiz M, Sáenz-Copete JC, Enríquez-Marulanda A, Ordóñez CA. Extra luminal migration of ingested fish bone to the spleen as an unusual cause of splenic rupture: Case report and literature review. Int J Surg Case Rep. 20I6;25:I84-7. doi: I0.IOI6/j.ijscr.2016.06.028

3. Bekkerman M, Sachdev AH, Andrade J, Twersky Y, Iqbal S. Endoscopic management of foreign bodies in the gastrointestinal tract: A review of the literature. Gastroenterol Res Pract. 2016;20I6:8520767. doi: IO.II55/20I6/8520767

4. Wang L, Wen W, Huang J, Hu W, Zhou R, Li X, et al. Endoscopic removal of a duodenal-perforating leg of 
glasses with dormia basket. Case Rep Gastroenterol. 20I6;IO:679-84. doi: I0.II59/000452205

5. Barragán C, Rueda JC, Espitia E, Uriza JL. Perforación intestinal por espina de pescado, revisión de la literatura científica y presentación de dos casos. Rev Colomb Cir. 20I2;27:79-84.

6. Tumay V, Guner OS, Meric M, Isik O, Zorluoglu A. Endoscopic removal of duodenal perforating fishbone - A case report. Chirurgia. 2015;IIO:47I-3.

7. Beecher SM, O'Leary DP, McLaughlin R. Diagnostic dilemmas due to fish bone ingestion: Case report and literature review. Int J Surg Case Rep. 20I5;I3:II2-5. doi: I0.IOI6/j.ijscr.20I5.06.034

8. Jiménez-Fuertes M, Moreno-Posadas A, Ruiz-Tovar J, Durán-Poveda M. Liver abscess secondary to duode- nal perforation by fishbone: Report of a case. Rev Esp Enferm Dig. 20I6;I08:44.

9. Jiménez RM, Flores M, Méndez C, Valera Z, López F, Pareja F. Perforation of the rectosigmoideal junction by ingestion of foreign bodies. Rev Esp Enferm Dig. 2009;IOI:88I-9.

Io. Fonseca-Sosa FK. Meckel's diverticulum perforated by a fishbone. An unusual presentation. Rev Esp Enferm Dig. 2017;I09:394-5. doi: I0.17235/reed.2017.4614/2016

II. Paixão TS, Leão RV, de Souza Maciel Rocha Horvat N, Viana PC, Da Costa Leite C, de Azambuja RL, et al. Abdominal manifestations of fishbone perforation: a pictorial essay. Abdom Radiol (NY). 2017;42:I087-95. 